OPEN ACCESS

Edited by:

Zhiye Chen,

People's Liberation Army General

Hospital, China

Reviewed by:

Sheng-Min Wang,

Catholic University of Korea,

South Korea

Zhao Dong,

Chinese PLA General Hospital, China

Douglas Greve,

Massachusetts General Hospital and

Harvard Medical School,

United States

*Correspondence:

Shuu-Jiun Wang

sjwang@vghtpe.gov.tw;

k123.wang@msa.hinet.net

${ }^{t}$ These authors have contributed equally to this work and share first authorship

*These authors have contributed equally to this work and share last authorship

Specialty section:

This article was submitted to Headache and Neurogenic Pain,

a section of the journal

Frontiers in Neurology

Received: 20 October 2021

Accepted: 07 January 2022

Published: 31 January 2022

Citation:

Wu J-W, Lai P-Y, Chen Y-L, Wang Y-F, Lirng J-F, Chen S-T, Lai K-L,

Chen W-T, Wu Y-T and Wang S-J (2022) The Use of Neuroimaging for

Predicting Sumatriptan Treatment Response in Patients With Migraine

Front. Neurol. 13:798695.

doi: 10.3389/fneur.2022.798695

\section{The Use of Neuroimaging for Predicting Sumatriptan Treatment Response in Patients With Migraine}

\author{
Jr-Wei Wu ${ }^{1,2+}$, Pi-Yi Lai ${ }^{3+}$, Yung-Lin Chen ${ }^{3}$, Yen-Feng Wang ${ }^{1,2}$, Jiing-Feng Lirng ${ }^{2,4}$, \\ Shu-Ting Chen ${ }^{2,4}$, Kuan-Lin Lai ${ }^{1,2}$, Wei-Ta Chen ${ }^{1,2}$, Yu-Te Wu ${ }^{3,5,6 \neq}$ and Shuu-Jiun Wang ${ }^{1,2,5 *}$ \\ ${ }^{1}$ Department of Neurology, Neurological Institute, Taipei Veterans General Hospital, Taipei, Taiwan, ${ }^{2}$ College of Medicine, \\ National Yang Ming Chiao Tung University, Taipei, Taiwan, ${ }^{3}$ Institute of Biophotonics, National Yang Ming Chiao Tung \\ University, Taipei, Taiwan, ${ }^{4}$ Department of Radiology, Taipei Veterans General Hospital, Taipei, Taiwan, ${ }^{5}$ Brain Research \\ Center, National Yang Ming Chiao Tung University, Taipei, Taiwan, ${ }^{6}$ Department of Biomedical Imaging and Radiological \\ Sciences, National Yang Ming Chiao Tung University, Taipei, Taiwan
}

Objectives: To identify the neuroimaging predictors for the responsiveness of patients to sumatriptan and use an independent cohort for external validation.

Methods: Structuralized headache questionnaire and 3-Tesla brain magnetic resonance imaging were performed in migraine patients. Regional brain volumes were automatically calculated using FreeSurfer version 6.0, including bilateral amygdala, anterior cingulated cortex, caudate, putamen, precuneus, orbitofrontal cortex, superior frontal gyri, middle frontal gyri, hippocampus, and parahippocampus. A sumatriptan-responder was defined as headache relief within $2 \mathrm{~h}$ after the intake of sumatriptan in at least two out of three treated attacks. We constructed a prediction model for sumatriptan response using the regional brain volume and validated it with an independent cohort of migraine patients.

Results: A total of 105 migraine patients were recruited, including 73 sumatriptan responders (69.5\%) and 32 (30.5\%) non-responders. We divided the migraine patients into derivation $(n=73)$ and validation cohorts $(n=32)$. In the derivation cohort, left hippocampal volume was larger in sumatriptan responders (responders vs. non-responders: $3,929.5 \pm 403.1$ vs. 3,611.0 $\pm 389.9 \mathrm{~mm}^{3}, p=0.002$ ), and patients with a larger left hippocampal volume had a higher response rate to sumatriptan (>4,036.2 vs. $\leq 4,036.2 \mathrm{~mm}^{3}$ : 92.0 vs. $56.3 \%, p=0.001$ ). Based on the findings, we constructed a prediction model using the cutoff value of $4,036.2 \mathrm{~mm}^{3}$, and we found that patients with a left hippocampal volume $>4,032.6 \mathrm{~mm}^{3}$ had a higher response rate to sumatriptan than those with a left hippocampal volume $\leq 4,032.6 \mathrm{~mm}^{3}$ (84.6 vs. $42.1 \%$, odds ratio $[\mathrm{OR}]=7.6[95 \%$ confidence interval $=1.3-44.0], p=0.013$ ) in the validation cohort.

Conclusion: Our study showed that left hippocampal volume is helpful to identify sumatriptan non-responders. This proof-of-concept study shows that left hippocampal volume could be used to predict the treatment response to sumatriptan in migraine patients.

Keywords: migraine, sumatriptan, hippocampus, prediction model, brain MRI 


\section{INTRODUCTION}

Migraine is a common and disabling neurological disorder that affects $9-15 \%$ of the general population (1-3). Currently, migraine treatment can be classified into acute and preventive therapies, and acute treatments can be categorized as migrainespecific and non-specific $(4,5)$. Triptans, which are $5-\mathrm{HT}_{1 \mathrm{~B} / 1 \mathrm{D}}$ receptor agonists, are widely used migraine-specific medications to abort acute migraine attacks (6). Even though generic products have emerged, sumatriptan is still the most widely prescribed acute treatment medication for migraine $(7,8)$. Additionally, clinical trials and post-marketing experience have shown its efficacy and tolerability since the introduction of sumatriptan in the 1990s $(9,10)$. According to current evidence and real-world experiences, $\sim 30 \%$ of migraine patients are non-responders to triptans, and individual responsiveness to triptans is variable (11). To date, the variability in the treatment response is not fully understood (12), and only a few studies have identified the predictors for triptan response in migraine. Current evidence showed that a lower pretreatment pain severity and a higher polygenic risk score were associated with a better response to triptans $(7,8)$. An early study suggested that triptans' efficacy is less optimal after a patient develops allodynia, but new controlled studies have shown conflicting results (9, 10). Regarding the neuroimaging predictors, no study directly identified structural or functional neuroimaging predictors for sumatriptan response in migraine. On the other hand, the neuroimaging predictor for preventive therapies for migraine has been identified. In chronic migraine, the iron deposition in the periaqueductal gray matter could be used for outcome prediction for onabotulinumtoxinA injection (11). Also, another study found responders to onabotulinumtoxinA injection have cortical thickening in the right primary somatosensory cortex, anterior insula, left superior temporal gyrus, and pars opercularis than non-responders (12). Currently, neuroimaging can be used to differentiate migraine from other primary headache disorders and certain brain regions associated with headache frequency, severity, and long-term outcomes after preventive therapies $(13,14)$. Hence, this proof-of-concept study hypothesized that neuroimaging could help predict the treatment outcomes of sumatriptan in migraine patients.

\section{METHODS}

We retrospectively analyzed the medical records, headache questionnaires, and neuroimaging of patients with migraine who visited the headache clinics of Taipei Veterans General Hospital (TVGH) between January 1, 2015, and December 27, 2017. The included patients should be able to complete the headache questionnaire, and the patient's medical records should be done by board-certificated neurologist specialized in headache medicine.

\section{Inclusion and Exclusion Criteria of Migraine Patients}

The inclusion criteria were as follows: (1). The patient's headache fulfilled the International Classification of Headache
Disorders (ICHD-3) criteria for migraine with or without aura, and the headache diagnosis was made by headache specialists; (2). Patients aged between 20 and 49 years; (3). Patients who completed the headache questionnaire; (4). Patients who had used sumatriptan to treat their migraine; (5). Patients who were able to report their treatment response to sumatriptan; and (6). Patients who were able to undergo magnetic resonance imaging (MRI) examinations without contraindications. The exclusion criteria were as follows: (1). Patients with underlying hypertension, diabetes mellitus, cerebrovascular diseases, epilepsy, or other neurodegenerative disorders; (2). Patients who had a history of traumatic brain injury or concussion; and (3). Patients who had been diagnosed with psychiatric disorders were excluded, including major depressive disorders, bipolar disorders, anxiety disorders, or schizophrenia.

\section{Measures of Sumatriptan Response}

All migraine patients participated in a semistructured interview at subsequent visits, which included questions about their response to sumatriptan, the timing of sumatriptan use, and usage of concomitant medications with sumatriptan. A sumatriptan responder was defined as patients with a decrease in headache intensity from moderate or severe to none or mild within $2 \mathrm{~h}$ after the intake of sumatriptan, in at least two out of three treated attacks (15-17). Patients with concomitant usage of acute medications other than sumatriptan were excluded from this study to ensure that the treatment responses came purely from sumatriptan.

\section{Headache Frequency and Severity}

In this study, we retrospectively analyzed items of the headache questionnaire, including headache frequency (headache days per month) and the Migraine Disability Assessment Score (MIDAS) questionnaire. The MIDAS questionnaire is widely used in clinical studies and controlled trials in headache medicine for analyzing migraine-related disability in a 3-months period (18, 19). The total score of the MIDAS questionnaire is the sum of five items, including the number of days of missed work/school, reduced productivity at work/school, missed household work, reduced productivity in household work, and missed family and/or social activities.

\section{Brain Neuroimaging}

All participants underwent whole-brain MRI using the same 3.0 T magnetic scanner (Discovery MR750 scanner, GE Healthcare, United States). Acquisition of T1-weighted images was based on 3D-FSPGR and AX-BRAVO sequences with the following parameters: repetition time $=9.384 \mathrm{~ms}$, echo time $=4.036 \mathrm{~ms}$, slice thickness $=1 \mathrm{~mm}$, flip angle $=12^{\circ}$, and matrix size $=256 \times 256 \times 172 \mathrm{~mm}^{2}$ using 3D-FSPGR protocol; repetition time $=9.184 \mathrm{~ms}$, echo time $=3.68 \mathrm{~ms}$, slice thickness $=1 \mathrm{~mm}$, flip angle $=12^{\circ}$, and matrix size $=256 \times$ $256 \mathrm{~mm}^{2}$ using AX-BRAVO protocol. Both 3D-FSPGR and AX-BRAVO were gradient-echo imaging sequences from GE Healthcare suitable for brain volume calculation, and regional brain volumes calculated from automated segmentation of 
T1-weighted structural images are reliable measures within the same scanner platform, even after upgrades (20).

\section{Structural Data Processing}

After imaging acquisition, preprocessing steps were conducted for better quality and creditability for subsequent analysis to measure the cortical morphological features. The first approach was to correct the head orientation to avoid any motion artifacts by making the AC-PC line congruent with the $\mathrm{y}$ axis by using ART (the acpcdetect program in automatic registration toolbox, https://www.nitrc.org/projects/art). All the images were resized to $1 \mathrm{~mm}^{3}$ isotropic voxel with a size of $256 \times 256 \times 256$. Second, bias field correction was performed to remove the inhomogeneity of images by using N4 Bias Field Correction in Advanced Normalization Tools (ANTs). Finally, skull stripping was performed by using HD-BET, which applies artificial neural networks as processing algorithms. Automated brain volume measurements were subsequently conducted using FreeSurfer version 6.0, which is open-source software for processing and analyzing human brain MRI images. The cortical volumes $\left(\mathrm{mm}^{3}\right)$ of the region of interest (ROIs) associated with migraine and analgesic effects were calculated, including the bilateral amygdala, anterior cingulate cortex, caudate, putamen, precuneus, orbitofrontal cortex, superior frontal gyri, middle frontal gyri, hippocampus, and parahippocampus $(13,21,22)$.

\section{Statistics}

Comparisons of demographics and clinical profiles between derivation and validation cohort were analyzed by using chisquare or $t$-tests as appropriate. Also, the differences in demographics and clinical profiles in responders and nonresponders were analyzed by using chi-square or $t$-tests as appropriate. In the derivation cohort, Bonferroni's correction for multiple comparisons was applied in the comparison of the 20 ROIs between responders and non-responders (corrected for 20 pairwise comparisons: $p<0.05 / 20=0.0025$ ). The significant variables were examined by using a classification and regression tree in order to obtain bivariate cutoff values for maximal sensitivity and specificity (23). A chi-square has been applied to compare response rates to sumatriptan between two sides of the cutoff value in the derivation and validation cohorts. The validation of the prediction model in both derivation and validation cohorts was considered exploratory; hence, we used $p$ $<0.05$ as the significance threshold. All statistical analyses were conducted with IBM SPSS (version 22.0).

\section{Ethics}

The study protocol was approved by the Institutional Review Board of Taipei Veterans General Hospital (2020-03-005AC).

\section{RESULTS}

\section{Demographics}

A total of 105 individuals with migraine (77 female and 28 male) were included in this study. Among them, 73 were sumatriptan responders (69.5\%) and 32 (30.5\%) were

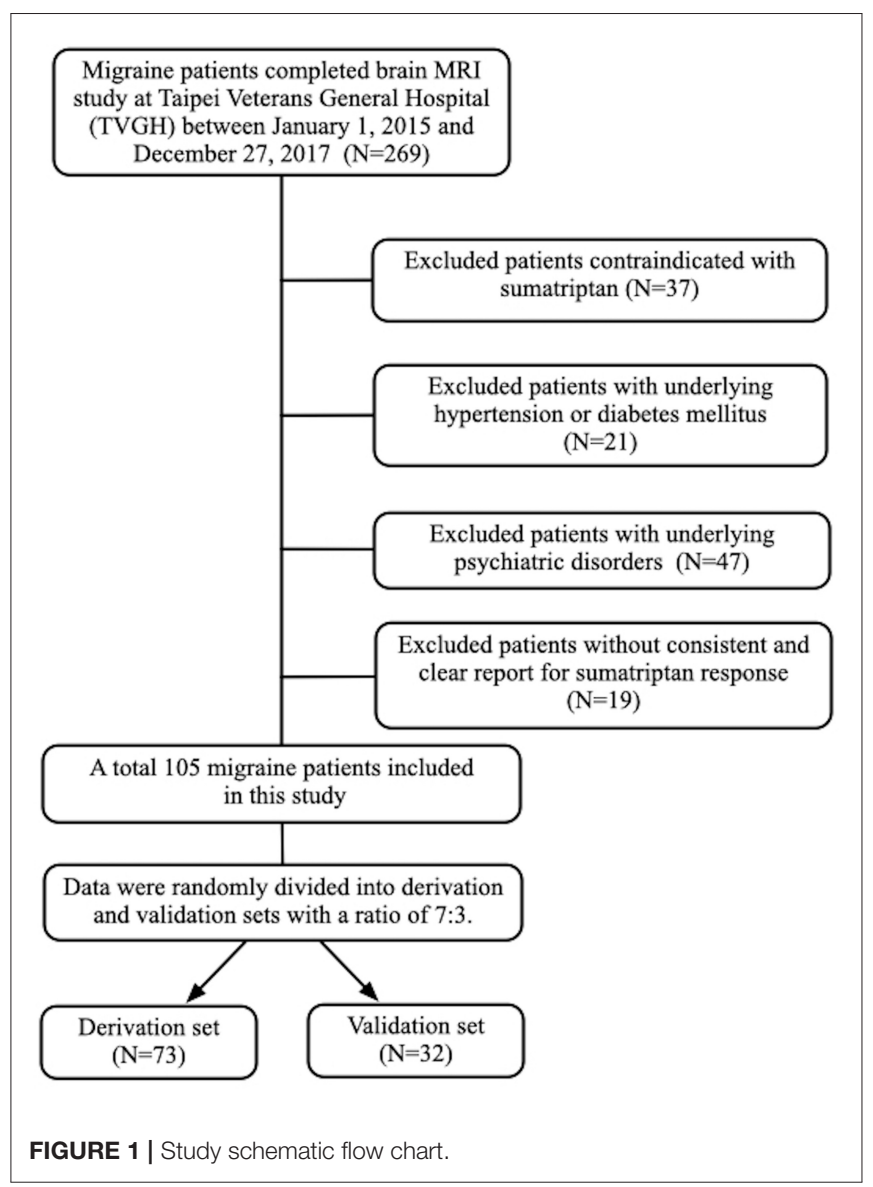

non-responders (Figure 1). The mean age of the study population was 33.2 (standard deviation $[\mathrm{SD}]=8.3]$ ) years. The prevalence of aura was $30.5 \%$, and chronic migraine (CM) accounted for $25.7 \%$ of the participants. The derivation and validation sets were randomly divided into at a ratio of $7: 3$, and there was no difference in demographics between the derivation and validation cohorts, as shown in Table 1.

\section{Potential Confounding Factors of Responders and Non-Responders}

Regarding the demographic factors, there are no differences between responders and non-responder in age (mean [SD] years for responders vs. non-responders: 33.4 [9.7] vs. 33.5 [8.7], $p=0.960$ ) or sex (responders: 12 males and 38 females; non-responders: 4 males and 19 females, $p=$ 0.529). Also, there were no differences in the clinical profiles between responders and non-responders, including prevalence of aura (responders vs. non-responders: 30.4 vs. $32.0 \%, p=$ $0.894)$, chronic migraine (CM) (responders vs. non-responders: 26.1 vs. $30.0 \%, p=0.732$ ), MIDAS (responders vs. nonresponders: 29.4 [32.3] vs. 27.8 [26.6], $p=0.839)$, or headache frequencies (mean [SD] headache days per month for responders vs. non-responders: 7.7 [7.1] vs. 8.4 [7.9], $p=0.678$ ) (Table 2). 
TABLE 1 | Demographics and descriptive statistics of potential confounding factors between the derivation and validation groups.

\begin{tabular}{lcc}
\hline Variables & $\begin{array}{c}\text { Derivation group } \\
(\mathbf{N}=\mathbf{7 3})\end{array}$ & $\begin{array}{c}\text { Validation group } \\
(\mathbf{N}=\mathbf{3 2})\end{array}$ \\
\hline Age, mean (SD), years & $33.4(9.3)$ & $32.6(7.8)$ \\
Sex, No. (\%) & & $12(37.5 \%)$ \\
Men & $16(21.9 \%)$ & $20(62.5 \%)$ \\
Women & $57(78.1 \%)$ & $9(28.1 \%)$ \\
Prevalence of migraine with aura & $23(31.5 \%)$ & $6(18.8 \%)$ \\
Prevalence of chronic migraine & $21(28.8 \%)$ & $8.4(5.3)$ \\
Headache frequency & $7.9(7.3)$ & $20.1(17.0)$ \\
MIDAS & $28.9(30.4)$ & $59.4 \%$ \\
Sumatriptan responder & $68.5 \%$ & 0.732 \\
Total intracranial volume & $1,537,205.7(149,894.7)$ & 0.284 \\
Sequence of brain MRI & & 0.736 \\
3D-FSPGR & $8(11.0 \%)$ & 0.130 \\
AX-BRAVO & $65(89.0 \%)$ & 0.370 \\
& & $0.846 .7(158,269.3)$ \\
\end{tabular}

${ }^{\star}$ Results were considered significant by $p<0.05$.

TABLE 2 | Demographics and clinical profiles of responders and non-responders in the derivation and validation group.

\begin{tabular}{|c|c|c|c|c|c|c|}
\hline \multirow[b]{2}{*}{ Variables } & \multicolumn{3}{|c|}{ Derivation group $(N=73)$} & \multicolumn{3}{|c|}{ Validation group $(N=32)$} \\
\hline & $\begin{array}{l}\text { Responders } \\
\qquad(N=50)\end{array}$ & $\begin{array}{l}\text { Non-responders } \\
\qquad(N=23)\end{array}$ & p value* & $\begin{array}{l}\text { Responders } \\
\qquad(N=19)\end{array}$ & $\begin{array}{c}\text { Non-responders } \\
\qquad(N=13)\end{array}$ & $p$ value ${ }^{\star}$ \\
\hline Age, mean (SD), years & $33.4(9.7)$ & $33.5(8.7)$ & 0.960 & $32.2(8.4)$ & $33.2(7.1)$ & 0.719 \\
\hline \multicolumn{7}{|l|}{ Sex, No. (\%) } \\
\hline Men & $12(24.0 \%)$ & $4(17.4 \%)$ & 0.529 & $9(47.4 \%)$ & $3(23.1 \%)$ & $0.170^{\star *}$ \\
\hline Women & $38(76.0 \%)$ & 19 (82.6\%) & & $10(52.6 \%)$ & $10(76.9 \%)$ & \\
\hline Prevalence of migraine with aura & $16(32.0 \%)$ & $7(30.4 \%)$ & 0.894 & $6(31.6 \%)$ & $3(23.1 \%)$ & $0.605^{\star \star}$ \\
\hline Prevalence of chronic migraine & 15 (30.0\%) & $6(26.1 \%)$ & 0.732 & $4(21.1 \%)$ & $3(23.1 \%)$ & $0.893^{\star \star}$ \\
\hline Headache frequency & $7.7(7.1)$ & $8.4(7.9)$ & 0.678 & $6.9(4.0)$ & $9.2(6.2)$ & 0.166 \\
\hline MIDAS & $29.4(32.3)$ & $27.8(26.6)$ & 0.839 & $17.8(12.2)$ & $23.4(13.7)$ & 0.23 \\
\hline Total intracranial volume, $\mathrm{mm}^{3}$ & $1,554,551.5(158,291.6)$ & $1,499,497.4(124,722.1)$ & 0.115 & $1,567,413.4(179,437.5)$ & $1,474,449.3(104,185.8)$ & 0.104 \\
\hline \multicolumn{7}{|l|}{ Sequence of brain MRI } \\
\hline 3D-FSPGR & $5(10.0 \%)$ & $3(13.0 \%)$ & 0.703 & $2(10.5 \%)$ & $1(7.7 \%)$ & 0.790 \\
\hline AX-BRAVO & 45 (90.0\%) & 20 (87.0\%) & & 17 (89.5) & 12 (92.3\%) & \\
\hline
\end{tabular}

${ }^{*}$ Results were considered significant by $p<0.05$.

${ }^{* *} p$-value calculated by linear-by-linear association.

\section{Regional Brain Volume and Sumatriptan Response (in the Derivation Cohort)}

Among the 20 ROIs, the left hippocampal volume was larger in the sumatriptan responders (responders vs. non-responders: 3,929.5 [403.1] vs. 3,611.0 [389.9] $\mathrm{mm}^{3}, p=0.002$ ) (Table 3). Using the classification and regression trees (CRT), we obtained a cutoff value of $4,036.2 \mathrm{~mm}^{3}$. By using the chi-square test, we found patients with a larger left hippocampal volume $(>4,036.2$ vs. $\leq 4,036.2 \mathrm{~mm}^{3}$ ) had a higher response rate to sumatriptan (92.0 vs. $56.3 \%, p=0.001)$ in the derivation cohort $(n=$ 73). We further explored the possible confounding effects on hippocampal values, and we found that hippocampal volume on both sides did not correlate with headache frequency (Left: Pearson's $r=0.069, p=0.561$; Right: Pearson's $r=0.107, p=$
0.368) or MIDAS (Left: Pearson's $r=0.052, p=0.664$; Right: Pearson's $r=0.189, p=0.110)$.

\section{Predicting Sumatriptan Response by Regional Brain Volume}

Based on the results from the derivation cohort $(n=73)$, we used a cutoff value of $4,032.6 \mathrm{~mm}^{3}$ to construct a prediction model by using a classification and regression tree (Figure 2). The validation cohort $(n=32)$, which had no differences in demographics or clinical profiles between the derivation and validation, has been used to examine the prediction model (Table 1). In the validation cohort (Figure 2), patients with a left hippocampal volume $>4,032.6 \mathrm{~mm}^{3}$ had a higher responder rate than those with a left hippocampal volume $\leq 4,032.6 \mathrm{~mm}^{3}$ 
TABLE 3 | GMV of ROls $\left(\mathrm{mm}^{3}\right)$ of responders and non-responders in the derivation group.

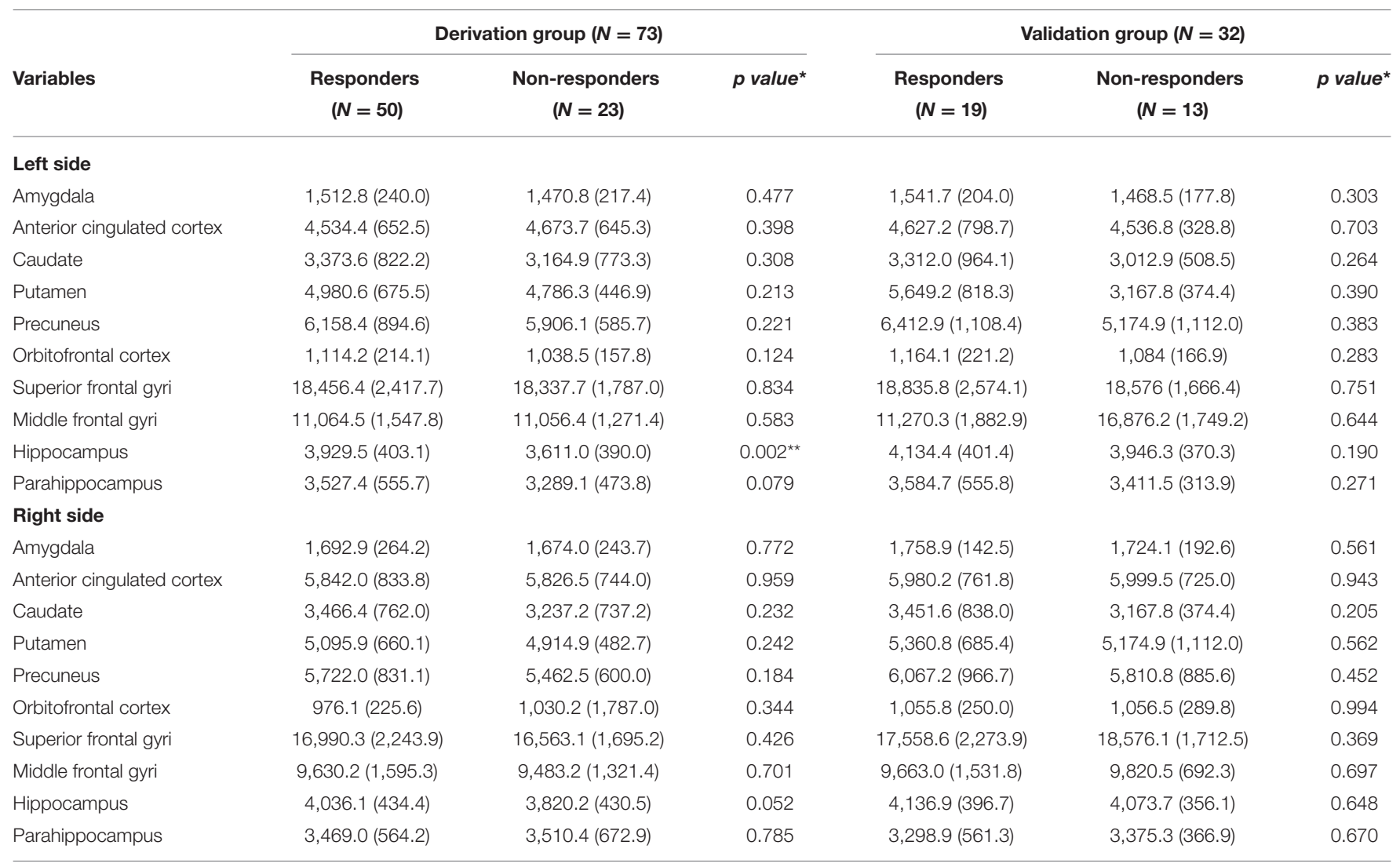

${ }^{*} p$-value calculated by $t$-test. ${ }^{* *}$ The results were considered significant by $p<0.05$.

$\left(>4,032.6\right.$ vs. $\leq 4,032.6 \mathrm{~mm}^{3}: 84.6$ vs. $42.1 \%$, odds ratio [OR] $=7.6[95 \%$ confidence interval $=1.3-44.0], p=0.013)$, with a high specificity and lower optimal sensitivity ( specificity $=84.6 \%$, sensitivity $=57.9 \%$, accuracy $=68.8 \%$ ).

\section{DISCUSSION}

This study found that sumatriptan responders have a larger left hippocampal volume than non-responders. When applying the prediction model to the independent validation cohort, patients with a left hippocampal volume $>4,032.6 \mathrm{~mm}^{3}$ had a higher responder rate than those with a left hippocampal volume $\leq 4,032.6 \mathrm{~mm}^{3}(\mathrm{OR}=7.6)$. The prediction model has a high specificity (84.6\%) but a lower optimal sensitivity (57.9\%). Instead of identifying good responders, the left hippocampal volume seems to be more suitable for identifying the "poor responders" to sumatriptan.

There are some studies that have aimed to identify predictors for the treatment response to triptans in migraine patients. One early study in 2004 found that pretreatment pain severity is a reliable predictor for the response to sumatriptan (7). Another recent study used genome-wide association studies and found a higher polygenic risk score for migraine associated with the sumatriptan response, which implies that a higher genetic burden of migraine is associated with a better response to migrainespecific treatment (8). To our knowledge, the present study identified left hippocampal volume as a new predictor for the response to triptans in migraine. However, the exact underlying mechanisms are unknown. One possible explanation is the direct effect of sumatriptan on the hippocampus. Although small amounts of triptan may cross the blood-brain barrier (BBB), sumatriptan has lower lipophilicity than other newer triptans (24). The relatively low brain penetration of sumatriptan is less likely to produce direct effects on the hippocampus (25). Additionally, a human postmortem brain study found that the distribution of sumatriptan-binding sites $\left(5 \mathrm{HT}_{1 \mathrm{D}}\right.$ receptor) is higher in the visual cortex, globus pallidus, and frontal cortex than in the hippocampus (26). Therefore, the association between the hippocampus and sumatriptan response seems unlikely to be attributed to the direct effect on the hippocampus.

The second explanation for our study findings is the "maladaptive theory." This hypothesis is supported by studies that found that patients with smaller hippocampal volumes may be more vulnerable or have maladaptation to stressful events (27). One brain perfusion study found the activation of the amygdala, brainstem, and hippocampus was associated with the analgesic effect of ibuprofen in tooth extraction, and these regions belong to the descending modulatory pathway (22). Another prospective study combined structural and functional MRI to analyze 

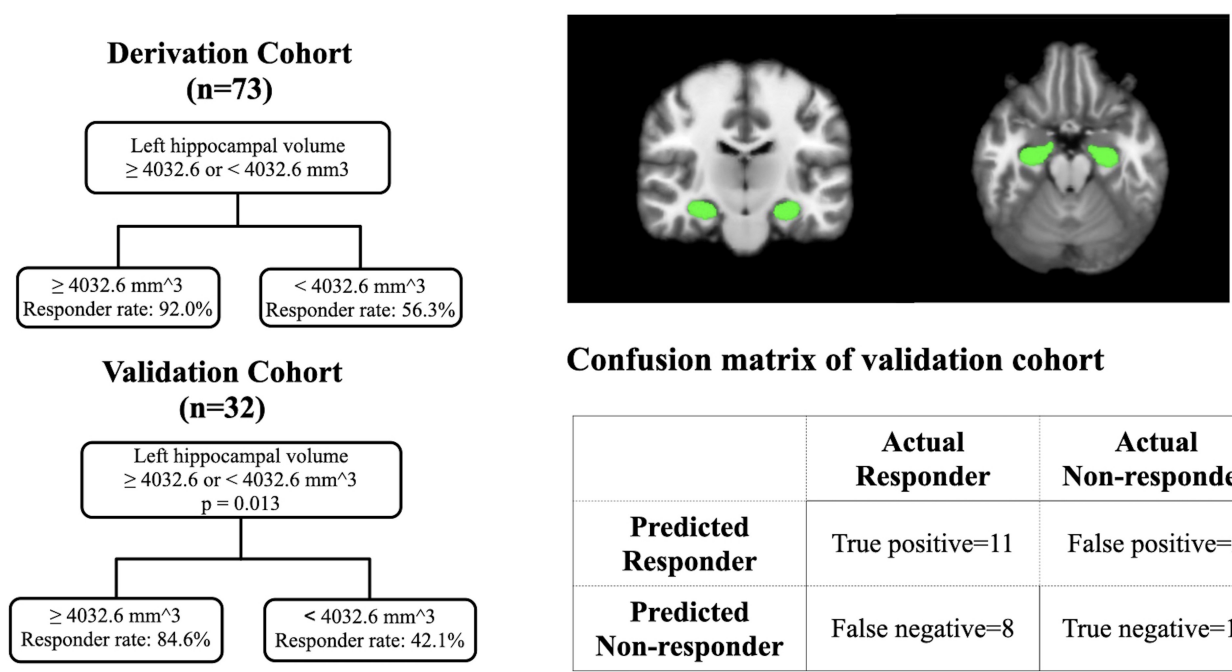

Confusion matrix of validation cohort

\begin{tabular}{|c|c|c|}
\hline & $\begin{array}{c}\text { Actual } \\
\text { Responder }\end{array}$ & $\begin{array}{c}\text { Actual } \\
\text { Non-responder }\end{array}$ \\
\hline $\begin{array}{c}\text { Predicted } \\
\text { Responder }\end{array}$ & True positive $=11$ & False positive $=2$ \\
\hline $\begin{array}{c}\text { Predicted } \\
\text { Non-responder }\end{array}$ & False negative $=8$ & True negative $=11$ \\
\hline
\end{tabular}

FIGURE 2 | The prediction model in the derivation cohort and validation cohort with the visualization of hippocampus.

patients with subacute back pain, which found that patients resistant to treatment have smaller amygdala and hippocampal volumes than those responsive to treatment (28). In menstrual pain, one study found that patients with a hippocampal volume associated with BDNF Val66Met polymorphisms and a smaller hippocampal volume had higher severity of menstrual pain (29). Additionally, studies found that patients with chronic pain conditions, (i.e., fibromyalgia, complex regional pain, and chronic low back pain) had smaller hippocampal volumes (30, 31). Regarding migraine, one study from our group found that a smaller hippocampal volume was associated with poor outcomes, indicating that the "maladaptive theory" could be applied to migraine patients (14). These findings suggest that there are reciprocal interactions between the hippocampus and pain; that is, individuals with an underlying smaller hippocampus may be more maladaptive to headaches or other pain conditions, less responsive to analgesics, and more vulnerable to developing chronic pain disorders. In this study, the "non-responders to sumatriptan" might be considered a maladaptive response to pain from a more vulnerable brain (32). The third explanation for the association between sumatriptan response and left hippocampal volume is the pain memory bias. One study could support this explanation, which found exaggerated remembered pain is not uncommon in patients with chronic low back pain. This phenomenon could be attributed to the shape displacement of the left posterior hippocampus (33). However, whether the biased pain memory could be analogized to the memory of analgesic response warrants further research and is beyond the scope of the present study.

The current study has limitations. First, the smaller hippocampal volume may be due to the aging process. Also, our study protocols did not include tests for cognitive function. Hence, the responsiveness to sumatriptan may have memory or recall bias. Nevertheless, the mean age of the present study population was $\sim 30$ years, which is an unlikely population to have cognitive deficits. Second, not responsive to one triptan, (i.e., sumatriptan) is not able to predict the response to other triptans (34), and further study is warranted to analyze the neuroimaging predictors of more than one acute medication for migraine. Third, our study excluded patients more than 50-year-old. Hence, our research findings could not represent the pediatric or elder population. The reason for selecting patients between 20 to 49 -year-old is to avoid the measurement of brain volume being confounded by the aging process. Also, migraine prevalence peaks from the age 20 s to 50s. The prediction model derived from this age range could represent most migraine patients in clinical settings (35). Fourth, our study design did not adjust for confounding factors, such as age, gender, intracranial volume, or ethnicity. The reason for not adjusting these factors is that our proof-of-concept study aimed to construct a prediction model easily applicable to the general population. Also, a recently-published review article addressed that there is no consensus for which and how many covariates should be adjusted for structural imaging studies and stated that "The current results highlight that the use of covariates has statistical and interpretative ramifications (36)." Fifth, the number of responders and non-responders is different in the derivation cohorts, and the imbalanced training dataset may cause overrepresentation of the majority class. On the other hand, our derivation and validation groups were based on data of consecutive patients, and the proportion of sumatriptan responders is usually higher than non-responders in the migraine population. The consecutive patients could prevent the possible confounding effect from the patient selection process.

\section{CONCLUSION}

This study found left hippocampal volume associated with the response to sumatriptan in migraine patients, and nonresponders tend to have smaller left hippocampal volume. 
According to the prediction model, patients with left hippocampal volume $>4,032.6 \mathrm{~mm}^{3}$ had a two-fold higher response rate than those $\leq 4,032.6 \mathrm{~mm}^{3}$ in an independent validation cohort.

\section{DATA AVAILABILITY STATEMENT}

The raw data supporting the conclusions of this article will be made available by the authors, without undue reservation.

\section{ETHICS STATEMENT}

The studies involving human participants were reviewed and approved by Institutional Review Board of Taipei Veterans General Hospital (2020-03-005AC). Written informed consent for participation was not required for this study in accordance with the national legislation and the institutional requirements.

\section{AUTHOR CONTRIBUTIONS}

J-WW, Y-TW, and S-JW had full access to all the data in the study and take responsibility for the integrity of the data and the accuracy of the data analysis. J-WW, P-YL, Y-TW, and S-JW: concept and design. J-WW, P-YL, S-TC, Y-LC, Y-TW, and J-FL: interpretation and analysis of neuroimaging. J-WW, Y-FW, K-LL, W-TC, and S-JW: treatment of all patients. J-WW and S-JW: drafting of the manuscript. J-WW: statistical analysis.

\section{REFERENCES}

1. Bigal ME, Liberman JN, Lipton RB. Age-dependent prevalence and clinical features of migraine. Neurology. (2006) 67:24651. doi: 10.1212/01.wnl.0000225186.76323.69

2. Wang SJ, Fuh JL, Young YH, Lu SR, Shia BC. Prevalence of migraine in Taipei, Taiwan: a population-based survey. Cephalalgia. (2000) 20:56672. doi: $10.1046 / j .1468-2982.2000 .00085 . x$

3. Ashina M. Migraine. (2020) 383:1866-76. doi: 10.1056/NEJMra1915327

4. Silberstein SD, Holland S, Freitag F, Dodick DW, Argoff C, Ashman E. Evidence-based guideline update: pharmacologic treatment for episodic migraine prevention in adults: report of the quality standards subcommittee of the american academy of neurology and the american headache society. Neurology. (2012) 78:1337-45. doi: 10.1212/WNL.0b013e3182535d20

5. Evers S, Afra J, Frese A, Goadsby PJ, Linde M, May A, et al. EFNS guideline on the drug treatment of migraine-revised report of an EFNS task force. Euro $J$ Neurol. (2009) 16:968-81. doi: 10.1111/j.1468-1331.2009.02748.x

6. Szperka CL, VanderPluym JH, Oakley CB. Pharmacologic acute and preventive treatment for migraine in children and adolescents. JAMA Neurol. (2020) 77:388-9. doi: 10.1001/jamaneurol.2019.4170

7. Christoph-Diener H, Ferrari M, Mansbach H. Predicting the response to sumatriptan. Sumatriptan Naratriptan Aggr Pat Database. (2004) 63:5204. doi: 10.1212/01.WNL.0000133207.70312.30

8. Kogelman LJA, Esserlind A-L, Francke Christensen A, Awasthi S, Ripke S, Ingason A, et al. Migraine polygenic risk score associates with efficacy of migraine-specific drugs. Neurol Genet. (2019) 5:e364-e. doi: 10.1212/NXG.0000000000000364

9. Burstein R, Collins B, Jakubowski M. Defeating migraine pain with triptans: a race against the development of cutaneous allodynia. Annal Neurol. (2004) 55:19-26. doi: 10.1002/ana.10786
J-WW, S-TC, Y-TW, and S-JW: obtained funding. Y-TW, J-FL, and S-JW: administrative, technical, or material support. Y-TW and S-JW: supervision. All authors critical revision of the manuscript for important intellectual content and acquisition, analysis, or interpretation of data. All authors contributed to the article and approved the submitted version.

\section{FUNDING}

This study was supported in part by grants from Taipei Veterans General Hospital [V108C-105 and V107C-135], the Ministry of Science and Technology of Taiwan [MOST110-2314-B-075-081, MOST 110-2314-B-075-035MY2, MOST 110-2321-B-010-005, MOST109-2314-B-075-002, MOST 108-2321-B-010-014-MY2, MOST 108-2321-B-010-001, and MOST 108-2314-B-010-023-MY3], the Ministry of Health and Welfare, Taiwan [MOHW 107-TDU-B-211-123001 and MOHW 108-TDU-B-211-133001], the Brain Research Center, and National Yang-Ming University from The Featured Areas Research Center Program within the framework of the Higher Education Sprout Project by the Ministry of Education (MOE) in Taiwan.

\section{ACKNOWLEDGMENTS}

We wish to thank the participants of the International Headache Academy (iHEAD) in 2019 for inspiring discussions during the meeting.

10. Cady RK, Freitag FG, Mathew NT, Elkind AH, Mao L, Fisher AC, et al. Allodynia-associated symptoms, pain intensity and time to treatment: predicting treatment response in acute migraine intervention. Headache. (2009) 49:350-63. doi: 10.1111/j.1526-4610.2009.01340.x

11. Domínguez Vivero C, Leira Y, Saavedra Piñeiro M, Rodríguez-Osorio X, Ramos-Cabrer P, Villalba Martín C, et al. Iron deposits in periaqueductal gray matter are associated with poor response to onabotulinumtoxina in chronic migraine. Toxins (Basel). (2020) 12:8. doi: 10.3390/toxins 120 80479

12. Hubbard CS, Becerra L, Smith JH, DeLange JM, Smith RM, Black $\mathrm{DF}$, et al. Brain changes in responders vs. non-responders in chronic migraine: markers of disease reversal. Front Hum Neurosci. (2016) 10:497. doi: 10.3389/fnhum.2016.00497

13. Chen WT, Chou KH, Lee PL, Hsiao FJ, Niddam DM, Lai KL, et al. Comparison of gray matter volume between migraine and "strict-criteria" tension-type headache. J Headache Pain. (2018) 19:4. doi: 10.1186/s10194-018-0834-6

14. Liu HY, Chou KH, Lee PL, Fuh JL, Niddam DM, Lai KL, et al. Hippocampus and amygdala volume in relation to migraine frequency and prognosis. Cephalalgia: Int J Headache. (2017) 37:1329-36. doi: 10.1177/0333102416678624

15. Cameron C, Kelly S, Hsieh SC, Murphy M, Chen L, Kotb A, et al. Triptans in the acute treatment of migraine: a systematic review and network metaanalysis. Headache. (2015) 55:221-35. doi: 10.1111/head.12601

16. Viana M, Genazzani AA, Terrazzino S, Nappi G, Goadsby PJ. Triptan nonresponders: do they exist and who are they? Cephalalgia. (2013) 33:8916. doi: 10.1177/0333102413480756

17. Tfelt-Hansen P, Pascual J, Ramadan N, Dahlöf C, D’Amico D, Diener $\mathrm{HC}$, et al. Guidelines for controlled trials of drugs in migraine: third edition. a guide for investigators. Cephalalgia: Int J Headache. (2012) 32:638. doi: $10.1177 / 0333102411417901$ 
18. Stewart WF, Lipton RB, Dowson AJ, Sawyer J. Development and testing of the migraine disability assessment (MIDAS) questionnaire to assess headache-related disability. Neurology. (2001) 56:S20-8. doi: 10.1212/WNL.56.suppl_1.S20

19. Silberstein S, Tfelt-Hansen P, Dodick DW, Limmroth V, Lipton RB, Pascual J, et al. Guidelines for controlled trials of prophylactic treatment of chronic migraine in adults. Cephalalgia. (2008) 28:484-95. doi: 10.1111/j.1468-2982.2008.01555.x

20. Jovicich J, Czanner S, Han X, Salat D, van der Kouwe A, Quinn B, et al. MRIderived measurements of human subcortical, ventricular and intracranial brain volumes: reliability effects of scan sessions, acquisition sequences, data analyses, scanner upgrade, scanner vendors and field strengths. Neuroimage. (2009) 46:177-92. doi: 10.1016/j.neuroimage.2009.02.010

21. Chong CD, Dumkrieger GM, Schwedt TJ. Structural co-variance patterns in migraine: a cross-sectional study exploring the role of the hippocampus. Headache. (2017) 57:1522-31. doi: 10.1111/head.13193

22. Hodkinson DJ, Khawaja N, O’Daly O, Thacker MA, Zelaya FO, Wooldridge $\mathrm{CL}$, et al. Cerebral analgesic response to non-steroidal anti-inflammatory drug ibuprofen. Pain. (2015) 156:1301-10. doi: 10.1097/j.pain.0000000000000176

23. Breiman L, Friedman J, Olshen R, Stone C. Cart. Classification and Regression Trees. Monterey, CA: Wadsworth and Brooks/Cole. (1984)

24. Dodick D, Martin V. Triptans and CNS Side-Effects:pharmacokinetic and metabolic mechanisms. Nature. (2004). 24:41724. doi: 10.1111/j.1468-2982.2004.00694.x

25. Tfelt-Hansen PC. Does sumatriptan cross the blood-brain barrier in animals and man? J Headache Pain. (2010) 11:5-12. doi: 10.1007/s10194-009-0170-y

26. Pascual J, del Arco C, Romon T, del Olmo E, Castro E, Pazos A. Autoradiographic distribution of [3h]sumatriptanbinding sites in post-mortem human. Brain. (1996) 16:31722. doi: 10.1046/j.1468-2982.1996.1605317.x

27. Lindgren L, Bergdahl J, Nyberg L. Longitudinal evidence for smaller hippocampus volume as a vulnerability factor for perceived stress. Cerebral Cortex. (2016) 26:3527-33. doi: 10.1093/cercor/bhw154

28. Vachon-Presseau E, Tétreault P, Petre B, Huang L, Berger SE, Torbey S, et al. Corticolimbic anatomical characteristics predetermine risk for chronic pain. Brain. (2016) 139:1958-70. doi: 10.1093/brain/aww100

29. Li WC, Chao HT, Lin MW, Shen HD, Chen LF, Hsieh JC. Neuroprotective effect of Val variant of BDNF Val66Met polymorphism on hippocampus is modulated by the severity of menstrual pain. NeuroImage Clinic. (2021) 30:102576. doi: 10.1016/j.nicl.2021.102576

30. Mutso AA, Radzicki D, Baliki MN, Huang L, Banisadr G, Centeno MV, et al. Abnormalities in hippocampal functioning with persistent pain. J Neurosci Offic J Soc Neurosci. (2012) 32:5747-56. doi: 10.1523/JNEUROSCI.0587-12.2012

31. McCrae CS, O'Shea AM, Boissoneault J, Vatthauer KE, Robinson ME, Staud $\mathrm{R}$, et al. Fibromyalgia patients have reduced hippocampal volume compared with healthy controls. J Pain Res. (2015) 8:47-52. doi: 10.2147/JPR.S71959

32. Tracey I. A vulnerability to chronic pain and its interrelationship with resistance to analgesia. Brain: J Neurol. (2016) 139:186972. doi: 10.1093/brain/aww147

33. Berger SE, Vachon-Presseau É, Abdullah TB, Baria AT, Schnitzer TJ, Apkarian AV. Hippocampal morphology mediates biased memories of chronic pain. Neuroimage. (2018) 166:86-98. doi: 10.1016/j.neuroimage.2017. 10.030

34. Dahlöf CG. Infrequent or non-response to oral sumatriptan does not predict response to other triptans-review of four trials. Cephalalgia: Int J Headache. (2006) 26:98-106. doi: 10.1111/j.1468-2982.2005.01010.x

35. Victor TW, Hu X, Campbell JC, Buse DC, Lipton RB. Migraine prevalence by age and sex in the United States: a life-span study. Cephalalgia. (2010) 30:1065-72. doi: 10.1177/0333102409355601

36. Hyatt CS, Owens MM, Crowe ML, Carter NT, Lynam DR, Miller JD. The quandary of covarying: a brief review and empirical examination of covariate use in structural neuroimaging studies on psychological variables. Neuroimage. (2020) 205:116225. doi: 10.1016/j.neuroimage.2019.1 16225

Conflict of Interest: The authors declare that the research was conducted in the absence of any commercial or financial relationships that could be construed as a potential conflict of interest.

Publisher's Note: All claims expressed in this article are solely those of the authors and do not necessarily represent those of their affiliated organizations, or those of the publisher, the editors and the reviewers. Any product that may be evaluated in this article, or claim that may be made by its manufacturer, is not guaranteed or endorsed by the publisher.

Copyright (C) 2022 Wu, Lai, Chen, Wang, Lirng, Chen, Lai, Chen, Wu and Wang. This is an open-access article distributed under the terms of the Creative Commons Attribution License (CC BY). The use, distribution or reproduction in other forums is permitted, provided the original author(s) and the copyright owner(s) are credited and that the original publication in this journal is cited, in accordance with accepted academic practice. No use, distribution or reproduction is permitted which does not comply with these terms. 